\title{
Segmentation of Brain MR Images with Directional Weighted Optimized Fuzzy C-Means Clustering
}

\author{
Arshad Javed \\ Faculty of Computer Science and Information \\ Al Jouf University \\ Saudi Arabia. \\ Faculty of Computer Science and Information Technology \\ Universiti Malaysia Sarawak \\ Malaysia. \\ arsh_qau@ju.edu.sa
}

\author{
Wang Yin Chai, Narayanan Kaluthramaiyer \\ Faculty of Computer Science and Information Technology \\ Universiti Malaysia Sarawak \\ Malaysia. \\ \{ycwang,nara\}@fit.unimas.my
}

\begin{abstract}
One of the fundamental and significant distinctiveness of an image is that, adjacent pixels are extremely correlated. The spatial information in the image improves the quality of clustering which is not utilized in the standard Fuzzy C-Means (FCM). FCM algorithm is not robust against noise. In this paper, we proposed an enhanced version of Fuzzy C-Means algorithm that incorporates spatial information into the membership function for clustering of brain MR images. The modified Fuzzy C-Means finds optimal clusters in an automatic way with the help of some cluster validity criteria. Additionally, spatial weighted information is incorporated in the spatial FCM. The spatial function is the weighted summation of the membership function in the neighborhood of each pixel under consideration. The advantages of this new method are: (a) it yields regions more homogeneous than those of other methods and (b) it removes noisy spots. It is less sensitive to noise as compared to other techniques. We tested our method on various brain MR images, and the technique has proved as a powerful method in the segmentation of noisy images.
\end{abstract}

\section{Keywords—directional weighted spatial FCM; segmentatio}

\section{INTRODUCTION}

The process of dividing a digital image into a number of non-overlapping and constituent regions each with distinct properties is referred as image segmentation. Separation of the Region of Interests (ROI) for the analysis purpose is the main task of the segmentation. Generally, tissue classes in the brain like White Matter (WM), Gray Matter (GM) and Cerebrospinal Fluid (CSF) constitutes to the region of interests. Whole pixels or objects in the region are similar with respect to some characteristics such as color, texture or intensity. Neighboring regions have significant dissimilarities with respect to the same characteristics. Variations in the composition of these tissue classes within specific regions or in the whole volume can be used to characterize disease severity.

Medical imaging is the main area of application of image segmentation used to find tumors, to measure tissue volumes, computer-guided surgery, a treatment arrangement and other pathology.

Since MR images contain the characteristics of uncertainty and ambiguity, hence fuzzy clustering segmentation has aroused comprehensive attenuation [1]. In the last decade, there have been proposed many different varieties of fuzzy clustering algorithms to solve the segmentation problem. FCM is the most popular fuzzy clustering algorithm.

The main objective of this study is to develop a mechanism to utilize the spatial information in the standard FCM algorithm to increase the performance of this.

\section{A. Magnetic Resonance Imaging (MRI)}

The speedy development of technologies like medical imaging now routinely allows living organs and organisms to be explored non invasively. Medical images for various applications are acquired that includes surgical simulation, image guided surgery, therapy evaluation and nano-science studies. While working with medical images, i.e. MRI, X-Ray, Computed Tomography (CT) and Ultrasound images, etc., it is frequent to delineate the areas and volumes of interest.

MRI is a well established diagnostic medical imaging technique that does not involve puncturing the skin or body and based on the nuclear magnetic resonance phenomenon [2]. MRI is used in the field of radiology to visualize the interior structure of the body. MRI supplies a deep information about soft tissue anatomy of the human brain. It uses radio waves and a magnetic field to construct detailed images of the structure of an organ. The resulting data from a MRI machine is shifted to a computer, which interprets the information and translates it into images. Echo Planer Imaging (EPI) is the most common technique used for the fast MRI. Fast and single-shots acquisitions make scanning very sensitive to magnetic susceptibility differences at air/tissue interfaces in the brain. Blurring effects and geometric distortions in EPI in spiral imaging caused by field inhomogeneity severely affect predictions about the brain of subject [3]. Due to these 